\title{
Competing many-body interactions in systems of trapped ions
}

\author{
A. Bermudez, ${ }^{1}$ D. Porras, ${ }^{1,2}$ and M. A. Martin-Delgado ${ }^{1}$ \\ ${ }^{1}$ Departamento de Física Teórica I, Universidad Complutense, 28040 Madrid, Spain \\ ${ }^{2}$ Max-Planck-Institut für Quantenoptik, Hans-Kopfermann-Strasse 1, 85748 Garching, Germany
}

(Received 22 January 2009; published 9 June 2009)

\begin{abstract}
We propose and theoretically analyze an experimental configuration in which lasers induce three-spin interactions between trapped ions. By properly choosing the intensities and frequencies of the lasers, three-spin couplings may be dominant or comparable to two-spin terms and magnetic fields. In this way, trapped ions can be used to study exotic quantum phases which do not have a counterpart in nature. We study the conditions for the validity of the effective three-spin Hamiltonian and predict qualitatively the quantum phase diagram of the system.
\end{abstract}

DOI: 10.1103/PhysRevA.79.060303

PACS number(s): 03.67.Ac, 37.10.Ty, 42.50.Wk, 75.10.Jm

Most theoretical models in condensed-matter physics rely on two-body interactions to describe a wide variety of phenomena, such as, for example, quantum phase transitions [1]. However, the presence of many-body interactions leads to exotic quantum effects, such as the existence of topologically ordered phases with anyonic excitations, which cannot be generally induced by pairwise couplings [2]. The beauty and complexity of those exotic models have motivated a good deal of recent theoretical work. Unfortunately, theory is still ahead of experimental implementations in this field since many-body interactions are usually negligible in most systems found in nature.

In this Rapid Communication, we show that three-body effective spin interactions may be implemented with trapped ions, thus opening a new avenue of research beyond the traditional paradigm of condensed-matter physics. The application of systems of trapped ions to the quantum simulation [3] of spin models has been theoretically analyzed, with a focus on conventional models with two-body interactions [4]. Furthermore, a recent proof-of-principle experiment has confirmed the validity of this idea [5]. By studying the implementation of three-spin couplings, we show here that trapped ions can also be used to engineer new quantum states of matter, with a phenomenology that does not have a counterpart in nature. Recently, three-body interactions have been theoretically analyzed in systems of ultracold atoms in triangular lattices [6] and cold polar molecules [7]. Our proposal would benefit from the advantages of experiments with trapped ions, such as measurement and manipulation at the single-particle level.

To sketch our idea, let us consider a system of spins and a spin operator $\sigma^{z}=\Sigma_{j} \sigma_{j}^{z}$. The spins are coupled to a bosonic mode $\left(H_{0}=\delta a^{\dagger} a\right)$ by both a linear displacement term, $H_{d}=F \sigma^{z}\left(a+a^{\dagger}\right)$, and a quadratic squeezing term, $H_{s}$ $=M \sigma^{z}\left(a^{2}+a^{\dagger 2}\right)$. After the adiabatic elimination of the mode, the lowest-order energy corrections include twobody $\left[\sim\left(F^{2} / \delta\right) \sigma_{j}^{z} \sigma_{k}^{z},\left(M^{2} / \delta\right) \sigma_{j}^{z} \sigma_{k}^{z}\right]$ and three-body $\left[\sim\left(M F^{2} / \delta^{2}\right) \sigma_{j}^{z} \sigma_{k}^{z} \sigma_{l}^{z}\right]$ interactions. In our scheme, this idea is realized in a system of ions in a linear array of microtraps $[8,9]$. The internal states of the ions play the role of the effective spins. Two off-resonant laser beams are tuned such that they induce linear and squeezing couplings between internal states and the collective vibrational modes of the chain. A third off-resonant beam induces an auxiliary linear coupling to partially cancel two-body interactions and, thus, to tune the relative strength of the two- and three-spin couplings. In this way, experiments may reveal quantum effects beyond the usual pairwise-induced correlations, such as a quantum tricritical point, and a quantum phase with a threebody order parameter.

We start by considering $N$ ions of mass $m$ and charge $e$, confined along a one-dimensional array of microtraps, with lattice spacing $a$ and trapping frequencies $\omega_{\alpha}$, with $\alpha$ $=x, y, z$ (see Fig. 1). We assume that the ions have two internal hyperfine ground-state levels $(|\uparrow\rangle,|\downarrow\rangle)$, and their motion can be accurately described in terms of collective quantized vibrations (i.e., phonons). Therefore, the system Hamiltonian $(\hbar=1)$ becomes

$$
H_{0}=-\sum_{j=1}^{N} h \sigma_{j}^{x}+\sum_{n=1}^{N} \sum_{\alpha=x, y, z} \Omega_{n \alpha} a_{n \alpha}^{\dagger} a_{n \alpha} .
$$

Here, $h$ is an effective magnetic field induced by a laser or microwave field coupled to the internal transition, $\sigma_{j}^{\alpha}$ are the Pauli matrices corresponding to each ion, $a_{n \alpha}^{\dagger}\left(a_{n \alpha}\right)$ are the phonon creation (annihilation) operators, and $\Omega_{n \alpha}=\omega_{\alpha}(1$ $\left.+c_{\alpha} \beta_{\alpha} \mathcal{V}_{n}\right)^{1 / 2}$ denotes the $n$th normal-mode frequency along the $\alpha$ axis. These frequencies are obtained by diagonalizing $\mathcal{V}_{n}=\Sigma_{j k} \mathcal{M}_{j n} V_{j k} \mathcal{M}_{k n}$, where $V_{j k}=\frac{1}{|j-k|^{3}}\left(1-\delta_{j k}\right)-\Sigma_{l \neq j} \frac{1}{|j-l|^{3}} \delta_{j k}$ is the Coulomb interaction in the limit of small vibrations, $\mathcal{M}_{j n}$ are the normal-mode wave functions, and $\beta_{\alpha}$ $=e^{2} / 4 \pi \epsilon_{0} m \omega_{\alpha}^{2} a^{3}, c_{x, y}=1$ and $c_{z}=-2$. Note that in the "stiff" limit $\beta_{\alpha} \ll 1$, the trapping potential is much larger than the Coulomb repulsion, and thus vibrational frequencies are restricted to a narrow band of width $\frac{1}{2} c_{\alpha} \beta_{\alpha} \omega_{\alpha}$ around the trapping frequencies $\omega_{\alpha}$ [see Figs. 2(a) and 2(b)].

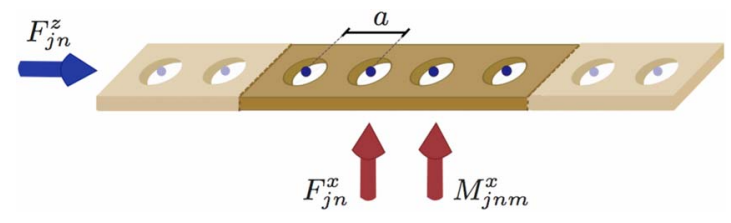

FIG. 1. (Color online) Linear array of microtraps subjected to three laser beams along the axial and radial directions. 

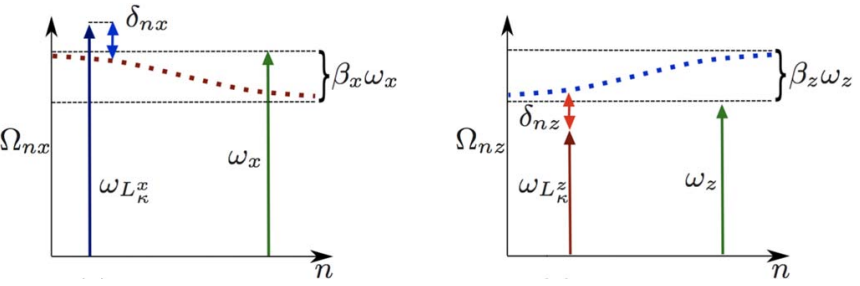

FIG. 2. (Color online) Phonon frequencies $\Omega_{n \alpha}$ of (a) radial and (b) axial modes in the stiff limit $\beta_{\alpha} \ll 1$, where the corrections due to the Coulomb energy $\frac{1}{2} c_{\alpha} \beta_{\alpha} \omega_{\alpha} \mathcal{V}_{n}$ have opposite signs for transverse $c_{x, y}=1$ or longitudinal $c_{z}=-2$ phonons. The coupling of spindependent dipole forces to vibrational modes is also shown (a) in the radial stiff limit, we can simultaneously blue detune every mode to the first sideband $\omega_{L_{1}^{x}} \gtrsim \Omega_{n x}$ or to the second sideband $\omega_{L_{2}^{x}}$ $\geq 2 \Omega_{n x}$. (b) In the axial stiff limit, every mode shall be red detuned to the first sideband $\omega_{L_{3}^{z}} \lesssim \Omega_{n z}$.

The ion chain will be subjected to three spin-dependent dipole forces $H_{I}=H_{L_{1}^{x}}+H_{L_{2}^{x}}+H_{L_{3}^{z}}$, such that the Hamiltonian becomes $H=H_{0}+H_{I}$. Here, the most general definition of a dipole force along direction $\alpha$ is

$$
H_{L_{\kappa}^{\alpha}}=\frac{\Omega_{L_{\kappa}^{\alpha}}}{2} \sum_{j=1}^{N}\left(e^{i\left(k_{L_{\kappa}^{\alpha}}^{\alpha} r_{j \alpha}-\omega_{L_{\kappa}^{\alpha}}^{\alpha t)}\right.}+\text { H.c. }\right) \sigma_{j}^{z},
$$

which is induced by a pair of lasers in a Raman configuration, such that $\omega_{L_{\kappa}^{\alpha}}$ is the detuning between the Raman beams, $\mathbf{k}_{L^{\alpha}}$ is the difference between the laser wave vectors [10], and $\Omega_{L_{\kappa}^{\alpha}}$ is the two-photon Rabi frequency [11]. The position of the ions in Eq. (2) can be expressed in terms of normal modes, $r_{j \alpha}=r_{j \alpha}^{0}+\sum_{n} \frac{\mathcal{M}_{j n}}{\sqrt{2 m \Omega_{n \alpha}}}\left(a_{n \alpha}^{\dagger}+a_{n \alpha}\right)$, where $\mathbf{r}_{j}^{0}=x_{0} \mathbf{e}_{x}$ $+j a \mathbf{e}_{z}$ are the ion equilibrium positions. Let us stress that in the stiff regime, $\beta_{\alpha} \ll 1$, it is possible to tune the dipole forces to a certain sideband of every normal mode of the ion chain and thus couples each spin to the whole ensemble of vibrational phonons.

The main effect of $H_{L_{1}^{x}}$ and $H_{L_{2}^{x}}$ is to induce conditional linear and squeezing terms, which will produce two- and three-spin couplings, respectively. This is achieved by tuning the dipole forces to the first and second blue sidebands $\left[\omega_{L_{1}^{x}} \gtrsim \Omega_{n x}\right.$ and $\omega_{L_{2}^{x}}=2 \omega_{L_{1}^{x}} \gtrsim 2 \Omega_{n x}$, see Fig. 2(a) $]$. Besides, the role of $H_{L_{3}^{z}}$ is to generate two-body couplings with an opposite sign as those induced by $H_{L_{1}^{x}}$ and thus to partially cancel two-spin interactions in favor of three-body effects. As discussed below, the optimal screening is achieved by red detuning this force to the first axial-sideband $\left[\omega_{L_{3}^{z}} \lesssim \Omega_{n z}\right.$, see Fig. 2(b)]. In the limit of resolved sidebands $\left|\delta_{n \kappa}^{\alpha}\right|=\mid \Omega_{n \alpha}$ $-\omega_{L_{\kappa}^{\alpha}} \mid \ll \Omega_{n \alpha}$ and weak couplings $\Omega_{L_{\kappa}^{\alpha}} \ll \Omega_{n \alpha}$, we get a timedependent interaction Hamiltonian, $H_{I}^{\kappa}(t)=H_{d}(t)+H_{s}(t)$, with

$$
\begin{gathered}
H_{d}(t)=\sum_{j, n} F_{j n}^{x}(t) a_{n x}^{\dagger} \sigma_{j}^{z}+\sum_{j, n} F_{j n}^{z}(t) a_{n z}^{\dagger} \sigma_{j}^{z}+\text { H.c. }, \\
H_{s}(t)=\sum_{j, n, m} M_{j n m}^{x}(t) a_{n x}^{\dagger} a_{m x}^{\dagger} \sigma_{j}^{z}+\text { H.c. }
\end{gathered}
$$

where we have introduced the coupling strengths $F_{j n}^{x}(t)$ $=i\left(\Omega_{L_{1}^{x}} \eta_{n 1}^{x} \sin \omega_{L_{1}^{x}} t+\Omega_{L_{2}^{x}} \eta_{n 2}^{x} \sin \omega_{L_{2}^{x}} t\right) \mathcal{M}_{j n} e^{i \phi_{x}}$ and $F_{j n}^{z}(t)$
$=i\left(\Omega_{L_{3}^{z}} \eta_{n 3}^{z} \sin \omega_{L_{3}^{z}} t\right) \mathcal{M}_{j n} e^{i \phi_{j z}}$ for the contributions arising from the first-sideband, and analogously $M_{j n m}^{x}(t)$ $=\frac{1}{2}\left(\Omega_{L_{2}^{x}} \cos \omega_{L_{2}^{x}} t\right) \eta_{n 2}^{x} \eta_{m 2}^{x} \mathcal{M}_{j n} \mathcal{M}_{j m} e^{i \phi_{x}}$ for the second sideband. Let us note that these couplings are switched on adiabatically and depend upon the laser intensities through $\Omega_{L_{\kappa}^{\alpha}}$, the Lamb-Dicke parameters $\eta_{n \kappa}^{\alpha}=k_{L_{\kappa}^{\alpha}} / \sqrt{2 m \Omega_{n \alpha}}$, and the relative phases $\phi_{j \kappa}^{\alpha}=k_{L_{\kappa}^{\alpha}} r_{j \alpha}^{0}$, assuming for simplicity $\phi_{j 1}^{x}=\phi_{j 2}^{x}$ $=\phi^{x}$.

To get the explicit form of the two- and three-spin couplings, we first note that $H_{0}+H_{d}(t)$ describes a set of forced harmonic oscillators. If we consider that dipole forces are adiabatically switched on and $t \gg 1 /\left(\Omega_{n \alpha}-\Omega_{L_{\alpha}^{\kappa}}\right)$, the evolution operator corresponding to $H_{0}+H_{d}(t)$ is $U_{d}(t)$ $=e^{-i t H_{0}} e^{-i t H_{\mathrm{eff}}^{(2)}} e^{S}$, with

$$
\begin{gathered}
H_{\mathrm{eff}}^{(2)}=\sum_{j>k} J_{j k}^{(2)} \sigma_{j}^{z} \sigma_{k}^{z}, \quad J_{j k}^{(2)}=\left(J_{j k}^{x}+J_{j k}^{z}\right), \\
J_{j k}^{\alpha}=\sum_{n \kappa \lambda}\left(\frac{\Omega_{L_{\kappa}^{\alpha}}}{2}\right)^{2} \frac{\left(\eta_{n \kappa}^{\alpha}\right)^{2} \mathcal{M}_{j n} \mathcal{M}_{k n}}{\Omega_{n \alpha}-\lambda \omega_{L_{\kappa}}},
\end{gathered}
$$

where $\lambda= \pm 1$ also considers nonresonant terms, and

$$
S=\sum_{n j \alpha} \xi_{j n}^{\alpha} a_{n \alpha}^{\dagger} \sigma_{j}^{z}-\text { H.c., } \quad \xi_{j n}^{\alpha}=i \int_{0}^{t} d \tau F_{j n}^{\alpha}(\tau) e^{i \Omega_{n \alpha} \tau} .
$$

Let us emphasize here that the effective two-spin interactions mediated by transverse $\left(J_{k J}^{x}>0\right)$ and longitudinal phonons $\left(J_{j k}^{z}<0\right)$ have opposite signs that interfere and lead to a partial screening of the two-body interactions. In this way, the effects of a three-body coupling, which arise as a consequence of the additional nonlinearities introduced by $H_{s}(t)$, can be experimentally accessed. Note that we are extending here the canonical transformation used in $[4,12]$ to the timedependent case, something that may find a broader application in the context of trapped ion quantum simulation. Working in the interaction picture with respect to $U_{d}$, the transformed Hamiltonian becomes $U_{d}^{\dagger} H_{s} U_{d}=H_{\mathrm{eff}}^{(3)}+H_{\mathrm{err}}$, where

$$
\begin{gathered}
H_{\mathrm{eff}}^{(3)}=\sum_{j>k>l} J_{j k l}^{(3)} \sigma_{j}^{z} \sigma_{k}^{z} \sigma_{l}^{z}, \\
J_{j k l}^{(3)}=\sum_{n m \lambda}\left(\frac{\Omega_{L_{1}^{x}}}{2}\right)^{2} \frac{\Omega_{L_{2}^{x}} \cos \phi_{x}}{2} \frac{\left(\eta_{n 1}^{x} \eta_{n 2}^{x}\right)^{2} \mathcal{M}_{j n} \mathcal{M}_{k n} \mathcal{M}_{j m} \mathcal{M}_{l m}}{\left(\Omega_{n x}-\lambda \omega_{L_{1}^{x}}\right)\left(\Omega_{m x}-\lambda \omega_{L_{1}^{x}}\right)},
\end{gathered}
$$

describes the effective three-spin interactions, and

$$
H_{\mathrm{err}}=\sum_{n m j k} M_{j n m}^{x}(t) \sigma_{j}^{z} a_{m x} e^{-i\left(\Omega_{n x}+\Omega_{m x}\right) t}\left(\begin{array}{c}
\delta_{j k} a_{n x}- \\
-2 \xi_{k n}^{x} \sigma_{k}^{z}
\end{array}\right)+\text { H.c. }
$$

are the residual nonresonant spin-phonon couplings [13].

The conditions under which these error terms become negligible, which are thoroughly described below, lead to a novel Hamiltonian in quantum magnetism 


$$
H_{\mathrm{eff}}=\sum_{j>k} J_{j k}^{(2)} \sigma_{j}^{z} \sigma_{k}^{z}+\sum_{j>k>l} J_{j k l}^{(3)} \sigma_{j}^{z} \sigma_{k}^{z} \sigma_{l}^{z}-h \sum_{j} \sigma_{j}^{x},
$$

where two- and three-body interactions contribute. We note here that naïve scalings $J^{(2)} \sim \eta^{2}$ and $J^{(3)} \sim \eta^{4}(\eta \sim 0.2$ in experiments) imply that two-spin couplings shall hinder three-spin effects. However, as shown in Eq. (4), the contribution to the two-body terms from $H_{L_{x}^{1}}\left(J_{j k}^{x}>0\right)$ is partially cancelled by that from $H_{L_{z}^{3}}\left(J_{j k}^{z}<0\right)$. This effect becomes optimal if the parameters of the axial dipole force are carefully chosen. In particular, $k_{L_{3}^{z}}$ should be an integer multiple of $2 \pi / a$, such that the ions sit on positions with the same relative phase of the dipole force. To achieve $J^{(2)} \sim J^{(3)}$, the laser must be red detuned, with an intensity $\Omega_{L_{z}^{3}}$ and detuning $\delta_{z}$ $<0$ fulfilling

$$
\begin{aligned}
& \left(\Omega_{L_{3}^{z}}^{2} k_{L_{3}^{z}}^{2}\right) f_{1}\left(\omega_{z}, \delta_{z}, \beta_{z}\right) \\
& \quad=\left(\Omega_{L_{1}^{x}}^{2} k_{L_{1}^{x}}^{2}\right) f_{2}\left(\omega_{x}, \delta_{x}, \beta_{x}\right),\left(\Omega_{L_{3}^{z}}^{2} k_{L_{3}^{z}}^{2}\right) f_{3}\left(\omega_{z}, \delta_{z}, \beta_{z}\right) \\
& \quad=\left(\Omega_{L_{1}^{x}}^{2} k_{L_{1}^{x}}^{2}\right) f_{3}\left(\omega_{x}, \delta_{x}, \beta_{x}\right)+\left(\Omega_{L_{2}^{x}}^{2} k_{L_{2}^{x}}^{2}\right) f_{4}\left(\omega_{x}, \delta_{x}, \beta_{x}\right),
\end{aligned}
$$

where the functions $f_{j}\left(\omega_{\alpha}, \delta_{\alpha}, \beta_{\alpha}\right)$ are listed in [14]. Furthermore, to obtain short-ranged spin interacting models, the radial stiffness parameter is restricted to $\beta_{\alpha} \sim 0.4 \delta_{\alpha} / \omega_{\alpha}$, where $\delta_{\alpha}=\omega_{\alpha}-\omega_{L_{\alpha}}$ is the detuning with respect to the bare trapping frequency. Under all these experimental constraints, at reach with current technology, we come to expressions for the many-body couplings

$$
J_{j k}^{(2)}=J_{2} \Lambda_{j k}, \quad J_{j k l}^{(3)}=\frac{1}{3} J_{3}\left(\Lambda_{j k} \Lambda_{k l}+\Lambda_{k j} \Lambda_{j l}+\Lambda_{j l} \Lambda_{l k}\right),
$$

where we have introduced the dipolar scaling function $\Lambda_{j k}$ $=1 /|j-k|^{3}$. The strength of these couplings is

$$
J_{2}=\frac{\beta_{x} F_{x}^{2}}{\left|\delta_{x}\right|}\left(1+\frac{\chi}{4}\right), \quad J_{3}=\frac{3 \beta_{x}^{2} \omega_{x}^{2} F_{x}^{2} M_{x} \cos \phi_{x}}{\left|\delta_{x}\right|^{4}},
$$

where $\chi=\beta_{z} \delta_{z} f_{2}\left(\omega_{z}, \delta_{z}, \beta_{z}\right) / \beta_{x}\left|\delta_{x}\right| f_{1}\left(\omega_{x}, \delta_{x}, \beta_{x}\right)$. We have introduced the bare linear and quadratic intensities by $F_{x}$ $=\frac{1}{2} \Omega_{L_{1}^{x}} \eta_{x}, M_{x}=\frac{1}{4} \Omega_{L_{2}^{x}} \eta_{x}^{2}$, and the bare Lamb-Dicke parameter $\eta_{x}=k_{L_{x}} / \sqrt{2 m \omega_{x}}$. Hence, the access to the wavelengths, detunings, and laser intensities leads to the controllability of twoand three-body couplings.

In order to check the viability of this proposal, we should carefully deal with the residual spin-phonon coupling in Eq. (7), which contains different nonresonant terms that contribute to the error with $O\left(M_{x}^{2} / \delta_{x}^{2}\right)[15]$ and $O\left(M_{x}^{2} F_{x}^{2} / \delta_{x}^{4}\right)$. Besides, the canonical transformation in Eq. (5) leads to an additional error that scales as $O\left(F_{x}^{2} / \delta_{x}^{2}\right)$ [16]. Hence, a feasible quantum simulation of competing many-body interactions requires the parameters $M_{x}$ and $F_{x}$ to be small in comparison to the laser detuning $\delta_{x}$. At this stage, we can check the viability of this proposal assuming the following available experimental parameters, which fulfill all the constraints above. Considering trapping frequencies $\omega_{x} \sim 10 \mathrm{MHz}$, laser detunings $\delta_{x} \sim 1.25 \mathrm{MHz}$, stiffness parameters $\beta_{x} \sim 0.05$, and setting the dipole force intensities to $F_{x} \approx M_{x} \sim 0.1 \delta_{x}$, we readily obtain an effective model of competing two- and three-spin interactions with $\left|J_{3}\right| \approx J_{2} \sim 0.6 \mathrm{kHz}$ and an error on the order of $E \sim 10^{-2}$. Furthermore, the modification of these parameters within the constraints detailed above allows us to experimentally access different regimes where $\left|J_{3}\right| / J_{2}$ $\lessgtr 1$ and thus observe the consequences of a competition between the many-body interactions in full glory. Let us note that one can also go beyond the usual dipolar regime in Eq. (10) by considering $\beta_{x} \omega_{x} / \delta_{x} \geq 0.5$. In this case, exotic longrange interactions between distant spins arise and offer a exceptional playground where the effects of the range of interactions can be studied. Additionally, the couplings can be raised to $\left|J_{3}\right| \approx J_{2} \sim 1-10 \mathrm{kHz}$. In order to achieve this interesting regime, one may relax the trapping frequencies or design the microtrap in such a way that the ion equilibrium distance is lowered.

The ability to independently tune the couplings $\left(J_{2}, J_{3}, h\right)$ offers the opportunity to study novel quantum phases of interacting spins. To get a qualitative picture, we consider the Hamiltonian where only the nearest-neighbor terms of the dipole couplings are kept

$$
H=J_{2} \sum_{j} \sigma_{j}^{z} \sigma_{j+1}^{z}+J_{3} \sum_{j} \sigma_{j}^{z} \sigma_{j+1}^{z} \sigma_{j+2}^{z}-h \sum_{j} \sigma_{j}^{x} .
$$

The ground state is determined by the competition between the different terms in Eq. (12): $J_{2}(>0)$ induces antiferromagnetic $(\mathrm{AF})$ order, $J_{3}(<0)$ will be shown to induce a novel ferrimagnetic $(\mathrm{F})$ phase, and $h(>0)$ encourages the system to lie in a disordered paramagnetic $(\mathrm{P})$ regime, where spins are aligned along the $x$ direction. We start our analysis by considering the following two limits:

(i) $J_{3}=0$ (two-spin quantum Ising model). This case is exactly solvable and shows a quantum phase transition at the critical coupling $J_{2}^{c}=h$ between the antiferromagnetic doubly degenerate ground state $\left(J_{2}>h\right) \quad\left|g_{\mathrm{AF}}\right\rangle$ $\in\{|\uparrow \downarrow \cdots \uparrow \downarrow\rangle,|\downarrow \uparrow \cdots \downarrow \uparrow\rangle\}$ and the paramagnetic phase $\left|g_{\mathrm{P}}\right\rangle$ $\propto \otimes_{j}\left(|\uparrow\rangle_{j}+|\downarrow\rangle_{j}\right)\left(J_{2}<h\right)[1]$. To quantify the degree of antiferromagnetic order, we define the order parameter $O_{\mathrm{AF}}(g)$ $=\frac{-1}{N-1} \sum_{j=1}^{N-1}\left\langle g\left|\sigma_{j}^{z} \sigma_{j+1}^{z}\right| g\right\rangle$, which fulfills $O_{\mathrm{AF}}\left(g_{\mathrm{P}}\right)=0$, and $O_{\mathrm{AF}}\left(g_{\mathrm{AF}}\right)=1$.

(ii) $J_{2}=0$ (three-spin quantum Ising model). In this case, three-spin interactions induce a novel quantum phase which can be fully characterized by the order parameter $O_{\mathrm{F}}(g)$ $=\frac{1}{N-2} \Sigma_{j=1}^{N-2}\left\langle g\left|\sigma_{j}^{z} \sigma_{j+1}^{z} \sigma_{j+2}^{z}\right| g\right\rangle$. The three-spin Ising model is no longer exactly solvable but shows self-duality properties [17], something that allows us to locate its critical point at the value $\left|J_{3}^{c}\right|=h$. This point separates a phase with a fourfold ferrimagnetic state, $\quad\left|g_{\mathrm{F}}\right\rangle \in\{|\uparrow \uparrow \uparrow \cdots \uparrow \uparrow \uparrow\rangle,|\uparrow \downarrow \downarrow \cdots \uparrow \downarrow \downarrow\rangle$, $|\downarrow \uparrow \downarrow \cdots \downarrow \uparrow \downarrow\rangle,|\downarrow \downarrow \uparrow \cdots \downarrow \downarrow \uparrow\rangle\} \quad\left(J_{3}>h\right)$, from the paramagnetic phase $\left(\left|J_{3}\right|<h\right)$. Remarkably, at $J_{3}^{c}$ there is a phase transition which belongs to $f$ the four-state Potts model universality class. Hence, three-spin correlations induce an exotic critical behavior different from the Ising universality class.

From these limiting regimes, one gets a notion of the complexity of the model for general $\left(J_{2}, J_{3}, h\right)$. In addition to the critical points studied above, the system should also hold a quantum phase transition between the AF and F phases, as well as a tricritical point, at which all the magnetic orders coexists [see Fig. 3(a)]. This qualitative picture is supported by the finite-size numerical calculations presented in Figs. 

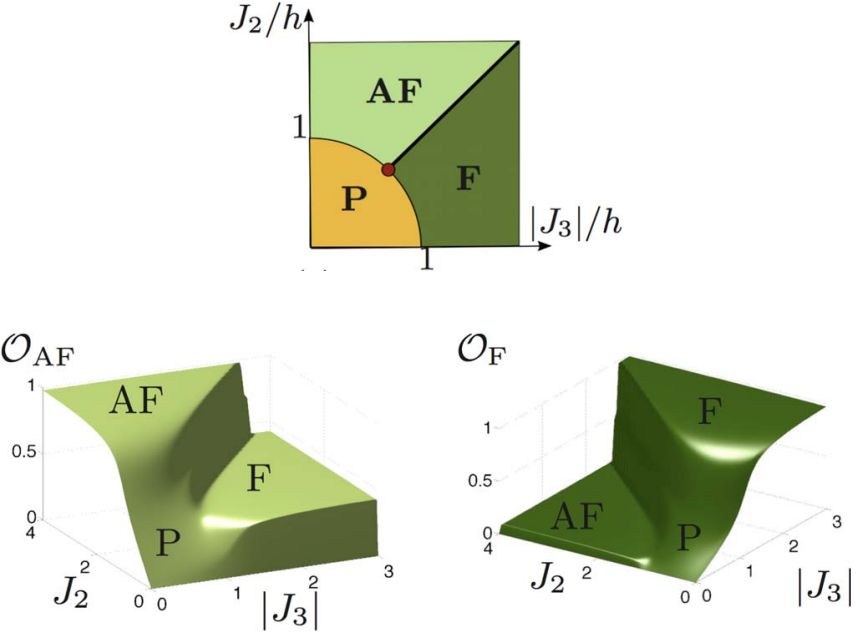

FIG. 3. (Color online) (a) Quantum phase diagram with P, AF, and $\mathrm{F}$ phases that coexist in the tricritical point (in red). Order parameters as a function of the couplings $J_{2}$ and $J_{3}$ for a chain with $N=15$ spins (b) antiferromagnetic order parameter (c) ferrimagnetic order parameter.

3(b) and 3(c). Note that the order parameters, $O_{\mathrm{AF}}$ and $O_{\mathrm{F}}$, may be measured by detecting the photoluminescence from individual ions, something that amounts to a quantum measurement of $\sigma^{z}$ [18]. It is precisely this ability of performing highly accurate measurements at the single-particle level, which allows us to characterize the full quantum phase diagram.

Let us briefly consider the implementation of these spin models using the more conventional linear Paul traps. Although the axial stiff limit cannot be achieved (i.e., the ion chain stability imposes $\left.\beta_{z} \gtrsim 1\right)$, it is still possible to devise effective two- and three-spin interactions. Focusing on the case of three ions, a similar procedure as that presented for microtraps, but considering a single mode in both radial directions $\alpha=x, y$ would yield

$$
H=J_{2}\left(\sigma_{1}^{z} \sigma_{2}^{z}+\sigma_{2}^{z} \sigma_{3}^{z}+\sigma_{3}^{z} \sigma_{1}^{z}\right)+J_{3} \sigma_{1}^{z} \sigma_{2}^{z} \sigma_{3}^{z}-h\left(\sigma_{1}^{x}+\sigma_{2}^{x}+\sigma_{3}^{x}\right),
$$

where there is full access to the different couplings $\left(J_{2}, J_{3}, h\right)$. In this case, we could even switch off the twobody term $J_{2}=0$ enhancing pure three-spin interactions and vice versa. In the same spirit as [5], one could perform a proof-of-principle experiment, where an initial separable paramagnetic state $|\mathrm{P}\rangle=|\rightarrow \rightarrow \rightarrow\rangle$ adiabatically evolves toward an entangled state with different types of ordering. In case we tune $J_{2}<0$ and $J_{3}=0$, the evolution would generate GHZ states $|\mathrm{GHZ}\rangle \sim|\uparrow \uparrow \uparrow\rangle+|\downarrow \downarrow \downarrow\rangle$, whereas for $J_{3}<0$ and $J_{2} \gtrsim 0$, one generates $W$ states $|W\rangle \sim|\uparrow \downarrow \downarrow\rangle+|\downarrow \uparrow \downarrow\rangle+|\downarrow \downarrow \uparrow\rangle$, studying thus the two nonequivalent classes of tripartite entanglement. Note that the Hamiltonians obtained with Paul traps are restricted to mean-field models, and microtraps should be used to implement strongly correlated systems.

In conclusion, we have shown that a system of trapped ions can be used to explore the singular phenomenology of spin models with three-body interactions. We have made a realistic experimental proposal, at reach with current technology, to access the peculiar phase diagram of an effective Hamiltonian with three-body interactions, characterized by the appearance of exotic phases without a counterpart in usual condensed-matter experiments.

A.B. and M.A.M.D acknowledge financial support from the Spanish MEC Project FIS2006-04885, CAM-UCM/ 910758, and the ESF Science Programme INSTANS 20052010. A.B. acknowledges support from a FPU MEC grant.
[1] S. Sachdev, Quantum Phase Transitions (Cambridge University Press, Cambridge, 1999).

[2] X. Wen, Quantum Field Theory of Many-body Systems (Oxford University Press, New York, 2004).

[3] R. Feynman, Int. J. Theor. Phys. 21, 467 (1982).

[4] D. Porras and J. I. Cirac, Phys. Rev. Lett. 92, 207901 (2004).

[5] A. Friedenauer et al., Nat. Phys. 4, 757 (2008).

[6] J. K. Pachos and M. B. Plenio, Phys. Rev. Lett. 93, 056402 (2004).

[7] H. P. Büchler, A. Micheli, and P. Zoller, Nat. Phys. 3, 726 (2007).

[8] J. Chiaverini and W. E. Lybarger, Jr., Phys. Rev. A 77, 022324 (2008).

[9] R. J. Clark, T. Lin, K. R. Brown, and I. L. Chuang, J. Appl. Phys. 105, 013114 (2009).

[10] D. Liebfried et al., Nature (London) 422, 412 (2003).

[11] The use of off-resonant walking waves, instead of standing waves [4], presents the advantage that there is no need to control the position of the ion chain with respect to the applied wave.
[12] C. Wunderlich, Laser Physics at the Limit (Springer, Heidelberg, 2001), p. 216.

[13] There is also an error term proportional to the transverse magnetic field $H_{\mathrm{err}}=2 i h \Sigma_{j n \alpha} \xi_{j n}^{\alpha} a_{n \alpha}^{\dagger} \sigma_{j}^{y}+$ H.c.. However, it gives a negligible contribution for the range of parameters that we are interested in $h \approx J_{2}, J_{3}$, which leads to insignificant corrections of the order $O\left(F_{x}^{6} / \delta_{x}^{6}\right)$.

[14] $f_{1}\left(\omega_{\alpha}, \delta_{\alpha}, \beta_{\alpha}\right)=\beta_{\alpha}^{2}\left(\omega_{\alpha}+\delta_{\alpha}\right) / \delta_{\alpha}^{3}, \quad f_{2}\left(\omega_{\alpha}, \delta_{\alpha}, \beta_{\alpha}\right)=\beta_{\alpha}^{2}\left(\omega_{\alpha}\right.$ $\left.+3 \delta_{\alpha} / 2\right) / 2 \delta_{\alpha}^{3}, \quad f_{3}\left(\omega_{\alpha}, \delta_{\alpha}, \beta_{\alpha}\right)=4 \beta_{\alpha} \omega_{\alpha}\left(\omega_{\alpha}-\delta_{\alpha}\right) / \delta_{\alpha}^{2}\left(2 \omega_{\alpha}-\delta_{\alpha}\right)^{2}$, $f_{4}\left(\omega_{\alpha}, \delta_{\alpha}, \beta_{\alpha}\right)=2 \beta_{\alpha} \omega_{\alpha}^{4} / \delta_{\alpha}^{2}\left(\omega_{\alpha}-2 \delta_{\alpha}\right)^{2}\left(3 \omega_{\alpha}-2 \delta_{\alpha}\right)^{2}$.

[15] In order to neglect the error term quadratic in phonon operators, the ion chain should be cooled down to $\bar{n}<0.1$, which does not require spectral resolution of the different modes (i.e., $\left.k_{\mathrm{B}} T \gg \beta \omega\right)$.

[16] X.-L. Deng, D. Porras, and J. I. Cirac, Phys. Rev. A 72, 063407 (2005).

[17] K. A. Penson, R. Jullien, and P. Pfeuty, Phys. Rev. B 26, 6334 (1982).

[18] D. Leibfried, R. Blatt, C. Monroe, and D. Wineland, Rev. Mod. Phys. 75, 281 (2003). 